\title{
ESTADO DE CONOCIMIENTO DE LAS AVES DE AGUAS CONTINENTALES DE CHILE
}

\section{SYNOPSIS OF THE INLAND AQUATIC BIRDS OF CHILE}

\author{
Pedro F. Victoriano ${ }^{1}$, Angélica L. González² \& Roberto Schlatter ${ }^{3}$ \\ ${ }^{1}$ Departamento de Zoología. Facultad de Ciencias Naturales y Oceanográficas. Universidad de Concepción. \\ Casilla 160-C. Concepción. Chile. Email: pvictori@udec.cl \\ ${ }^{2}$ Centros de Estudios Avanzados en Ecología y Biodiversidad. CASEB. Departamento de Ecología. \\ Facultad de Ciencias Biológicas. Pontificia Universidad Católica de Chile. Santiago de Chile. \\ ${ }^{3}$ Laboratorio de Ornitología. Instituto de Zoología. Universidad Austral de Chile. Valdivia. Chile
}

\begin{abstract}
RESUMEN
En este trabajo se informa de manera sintética la composición de especies, distribución, estatus de conservación y algunos aspectos de la biología de las aves acuáticas de ambientes continentales de Chile. Esta avifauna está compuesta por un total de 133 especies, distribuidas en 69 géneros, 21 familias y 10 órdenes. El grupo representado por el mayor número de especies es el Orden Charadriiformes (51 especies), con una alta presencia en ambientes ecotonales estuarinos. En ambientes de aguas interiores el grupo con más especies es el Orden Anseriformes (29 especies). La distribución de la riqueza de especies se corresponde en términos generales con las tendencias de representación para cada Orden en Sudamérica. De acuerdo a esta revisión, una parte importante de los registros para Chile son esporádicos (28 especies) o visitantes regulares (13 especies), y sólo un $69 \%$ del total pueden ser consideradas como residentes de nuestra avifauna acuática. De acuerdo a los estados de conservación, un total de 25 especies es considerada en algún sistema de clasificación, de las cuales una especie, Numenius borealis o zarapito boreal, se considera extinta en todo su rango distribucional. Según los registros de los últimos 50 años, el pidén austral (Rallus antarcticus) es una especie extinta en la zona central de Chile, quedando sólo algunas poblaciones poco abundantes en el extremo sur del país y en el sur de Argentina. En Chile no existen especies de aves acuáticas endémicas. Sin embargo, al considerar como áreas de análisis las regiones biogeográficas clásicas de nuestro país, existen dos zonas con un alto número de especies exclusivas: en la ecorregión Desértica 9,1\% del total nacional son endémicas de esa zona, mientras que la Tropical o Puna incluye un 7,6\%. A pesar de que en las ecorregiones Mediterránea y Oceánica existe un alto número de especies, éstas muestran pocos taxa propios (3\% y 2,3\% respectivamente). Se discute la información entregada en torno a aspectos distribucionales, ecológicos y de los aspectos poco estudiados sobre las aves acuáticas chilenas.
\end{abstract}

PALABRAS Claves: Chile, aves acuáticas, humedales, riqueza taxonómica.

\section{ABSTRACT}

As an overview, this article presents the species composition, distributions, conservation status and some aspects of biology of aquatic birds from Chile. This avifauna is constituted by 133 species, sorted in 69 genera, 21 families and 10 orders. The group represented by the higher number of species is the Order Charadriiformes (51 species), with a high proportion of species inhabiting estuarine ecotones. In freshwater environments the group with the higher richness is the Order Anseriformes (29 species). The distribution of the species richness is concordant with the trends of representation for each Order in South America. According to this revision, great part of registers for Chile are sporadics (28 spp) or regular migrants (13 spp), and only the $69 \%$ of the total can be considered residents in Chile. According to the conservation status, a total of 25 species is considered in some system of classification, of which a species, Numenius borealis (esquimo curlew), is extinct. According to registries of lasts 50 years, the austral rail (Rallus antarcticus) is an extinct species in Central Chile, being represented by few populations in Southern Chile and Argentina. In Chile endemic species of aquatic birds do not exist. Nevertheless, when considering like analysis areas the Chilean ecoregions, exist two zones with a high number of exclusive species: the Desertica ecoregion with $9.1 \%$ endemism, and Tropical or Puna with a 7.6\% of endemic species. Although in the Mediterranea and Oceánica ecoregions exists a high number of species, these show few exclusive taxa (3\% and $2.3 \%$ respectively). The distributional, ecological and poorly studied aspects on the Chilean aquatic birds are discussed.

KEYwords: Chile, wetlands, aquatic birds, taxonomic richness. 
Aves de aguas continentales de Chile: Victoriano, P. et aL.

\section{INTRODUCCION}

El grupo de vertebrados terrestres de mayor riqueza específica en Chile es el de las aves. Dentro de esta Clase, las de ambientes acuáticos continentales conforman aproximadamente el $29 \%$ de las 462 especies registradas para el país (Araya \& Millie 1996). Aunque por sus atributos de alta vagilidad este grupo no presenta especies endémicas para Chile, su riqueza y singularidad lo hacen un grupo importante en la estructuración de los ecosistemas de humedales. El estudio científico de las aves de Chile se inició con L. Landbeck en 1852 y R. A. Philippi en 1853. El aporte de este último fue revisar críticamente el estudio de Molina (1782) con lo cual surge el primer texto recopilatorio, actualmente considerado un clásico de la literatura ornitológica chilena (Philippi 1864). Goodall et al. en 1946 y 1951 publicaron respectivamente en dos volúmenes un nuevo texto que incluye a toda la avifauna chilena, y posteriormente Johnson $(1965,1967)$ publicó también en dos volúmenes, pero en una versión en inglés, un texto que incluía la totalidad de la avifauna chilena. Posterior a estos trabajos, el estudio de las aves en nuestro país estuvo por mucho tiempo remitido fundamentalmente a informar la presencia de especies en sitios geográficos y sólo en las ultimas dos décadas se han agregado trabajos que tratan aspectos más detallados como por ejemplo ecología y comportamiento (Kennedy 1977, Riveros et al. 1981, Vilina 1995). La situación taxonómica de nuestra avifauna a nivel específico parece haber llegado a una fase estable, pero el grado de conocimiento sobre aspectos biológico-evolutivos es aún bastante pobre. Gran parte de los antecedentes han provenido de aficionados ("birding"), quienes se han dedicado a entregar antecedentes sobre la avifauna acuática, lo cual sin duda ha sido un gran aporte. A nivel del continente americano se han publicado importantes textos recopilatorios que incluyen a las especies presentes en Chile. Por ejemplo, los textos de Ridgely \& Tudor (1989 y 1994), en dos volúmenes, considera solo paseriformes, y de manera muy parcial a especies acuáticas chilenas. El volumen IV, aún no publicado de esta serie programada, incluirá exclusivamente las especies acuáticas de Sudamérica. En este mismo sentido la obra de De la Peña \& Rumboll (1998) constituye un buen texto para todas las especies del sur de Sudamérica, incluyendo la mayor parte de las es- pecies acuáticas registradas para Chile. Dichos textos están dirigidos fundamentalmente para trabajos de identificación de especies con alguna información sobre su biología y distribución. Los textos modernos que tratan exclusivamente aves chilenas, y por ende las acuáticas, son pocos. Hasta ahora uno de los más utilizados a modo de manual de campo es el texto de Araya \& Millie (1996), y sólo recientemente han surgido textos un poco más elaborados desde el punto de vista estético y de información comprimida (v.g. Jaramillo 2003; Martínez \& González 2004). Otros textos consideran exclusivamente especies acuáticas de Chile (e.g. Figueroa et al. 2001; Rottmann 1995).

Debido a su alta vagilidad, las aves muestran peculiaridades respecto a la situación de otros grupos menos móviles, lo que, por lo demás, explica el bajo porcentaje de endemismos chilenos. Esto hace también más dinámica su situación geográfica, generando un margen de error mayor en la delimitación de las áreas distribucionales por especie, y de todas las estadísticas derivadas. Esto obliga a ponderar de alguna manera la composición avifaunística chilena, para la cual debe considerarse la condición de ave residente o visitante según sea su persistencia o situación reproductiva dentro del territorio nacional. Se sabe de la ocurrencia de un número importante de especies que sólo han sido registradas en escasas ocasiones para Chile, por lo que corresponden a taxa atípicos y tal vez de distribución marginal dentro de la avifauna acuática nacional. Por ejemplo, tres especies de garzas sólo han sido avistadas en contadas ocasiones y sólo en el extremo norte de Chile (Egretta caerulea o garza azul, E. tricolor o garza tricolor y E. sacra o garza de arrecife). Para cualquier análisis zoogeográfico, la condición de especie residente, visitante o esporádica es un aspecto relevante.

En cuanto a la singularidad y fragilidad de la avifauna acuática, es sabido por una parte que los ambientes de humedales reúnen en espacios reducidos una alta riqueza de especies, en su mayoría aves especializadas y altamente sensibles a cambios ambientales (Tabilo et al. 1996). Esto se suma al hecho de que los ambientes de humedales frecuentemente son considerados como sitios sustituibles para otros fines, como por ejemplo rellenos para uso habitacional, lo que los expone a una alta tasa de deterioro o desaparición (Muñoz \& Möller 1997). Actualmente no existe un trabajo que incorpore en 
un listado actualizado y que incluya algunos criterios ecológicos a las especies de aves acuáticas continentales de Chile. En este trabajo entregamos una síntesis en este sentido, junto con recopilar sus estados de conservación y las tendencias distribucionales y de riqueza en Chile. El objetivo de este trabajo es reunir esta información como una manera de contribuir al incremento de los estudios futuros sobre aves acuáticas de aguas interiores chilenas.

\section{MÉTODO}

La composición taxonómica base y los rangos distribucionales se obtuvieron de fuentes bibliográficas, tanto específicas para Chile (e.g. Araya et al. 1995 y Araya \& Millie 1996), como de mayor cobertura geográfica pero que incluyen a las especies presentes en el país (De la Peña \& Rumboll 1998; Canevari et al. 2002). El criterio para incluir las especies en los listados fue su consideración en el censo neotropical de aves acuáticas (Espinosa 2000), o por su clara dependencia de hábitats de humedales. Parte de la información fue extraída también desde sitios de la red, como es el caso de los sistemas de clasificación internacional de categorías de conservación de la IUCN y CITES ${ }^{1,2}$. Dos criterios de categorías de conservación para Chile fueron considerados; uno a nivel nacional (Glade 1989), y otro exclusivo para aves acuáticas para la Región del BíoBío (CONAMA 1999). Las especies de aves fueron clasificadas según la frecuencia de registros en el país en: E: esporádicas (uno o dos registros locales), V: visitantes regulares (migratorias anuales), y $\mathrm{R}$ : residentes (presentes todo el año). De acuerdo al tipo de ambiente que frecuenta cada especie, éstas fueron clasificadas en I: habitantes exclusivos de aguas interiores, y ES: especies de ambientes ecotonales como estuarios o desembocaduras de ríos. Las especies generalistas en este sentido fueron consignadas como pertenecientes a ambos grupos.

Con el fin de sintetizar el aporte de cada grupo de aves a la diversidad de especies del país, hemos estimado conveniente ponderar el número de familias, géneros y especies contenidas al interior de cada Orden. Considerando la lógica de que los grupos filogenéticamente más heterogéneos debieran aportar en mayor grado a la diversidad total de cualquier comunidad, es importante considerar no sólo la participación

\footnotetext{
${ }^{1}$ http://www.redlist.org/

${ }^{2}$ http://www.cites.org/
}

del número de especies, sino también de las jerarquías taxonómicas supraespecíficas. Si bien nuestra realidad dista de contar con información filogenética para todos los grupos de aves, se puede hacer una aproximación burda al ponderar diferencialmente las riquezas de diferentes jerarquías taxonómicas. La complejidad filogenética al interior de cualquier grupo será mayor según sea la suma total de los cambios evolutivos que han ocurrido al interior de éste, y por ende se correlaciona con la longitud total de las ramas de un árbol (Felizola 2004). Tal complejidad debiera aumentar en forma exponencial a medida que pasamos desde una jerarquía menor (e.g. géneros) a una inmediatamente superior (e.g. familias) (Avise 2004). Por lo anterior, hemos considerado razonable usar un índice que integra de manera ponderada la participación desde especie a familia al interior de cada Orden. Este índice de Riqueza Taxonómica Ponderada (Rtp) es como se detalla a continuación:

$R t p=\frac{4 F+2 G+1 S p}{7}$

donde $F$ es el número de familias, $G$ es el número de géneros y $S p$ el de especies al interior de cada Orden. Matemáticamente este índice puede adquirir valores desde 1 (riqueza ponderada mínima, correspondiente a una sola especie, y por ende un género y una sola familia, hasta infinito. Si bien este índice es aplicable tanto para comparaciones entre áreas como entre taxa superiores, en este caso sólo lo aplicamos a cada Orden de aves. Para fines del análisis zoogeográfico, se consideraron como áreas de trabajo a las ecorregiones generales de Chile propuestas por Di Castri (1968). Estimamos adecuado esto último debido a la gran escala de dispersión de las aves. De esta forma se consideraron las ecoregiones Desértica (litoral + interior), Tropical (marginal + de altura), Mediterránea (sensu lato), Andina, Oceánica (sensu lato) y Oceánica trasandina (extremo sur del país con influencia patagónica). A partir de los datos de presencia/ausencia de cada especie de ave en cada ecorregión, determinamos sus endemismos y calculamos el índice de Jaccard, el que adquiere valores de cero para comparaciones de áreas que no comparten especies, a 1 para pares con composición específica idéntica. A partir de la matriz de similitud obtenida se construyó un dendrograma mediante UPGMA. 
Aves de aguas continentales de Chile: Victoriano, P. et aL.

\section{RESULTADOS Y DISCUSION}

\section{COMPOSICIÓN, RIQUEZA TAXONÓMICA Y ESTATUS}

En Chile se ha registrado un total de 132 especies de aves que habitan en aguas interiores o en ambientes ecotonales mar-agua dulce (Tabla I; Anexo I), constituyendo un porcentaje importante $(29 \%)$ del total de especies del país. Este porcentaje se reparte en 10 órdenes y entre ellos, el mejor representado es el de los caradriformes (playeros, becacinas y gaviotas) con 51 especies. Le siguen en cuanto al número de especies los órdenes Anseriformes (patos y gansos), con 29, y Ciconiiformes (garzas y bandurrias) con 18. Estas cifras concuerdan en términos generales con las obtenidas para el índice Rtm, donde el valor más alto se obtuvo para los caradriformes, sugiriendo que éste es el grupo más heterogéneo desde un punto de vista taxonómico. De los otros dos grupos con mayor riqueza, los ciconiformes, aunque están representados por menos especies que los anseriformes, tienen un valor de Rtm levemente mayor. Esto quiere decir que, aunque alberga un menor número de especies, los ciconiformes son más heterogéneos taxonómicamente que el grupo de los patos y gansos de Chile. En términos generales podemos inferir de manera aproximada que, entre las aves de aguas continentales chilenas, estos tres órdenes constituirían los grupos filogenéticamente más diversos. Un cuarto grupo con una alta riqueza taxonómica ponderada es el de los paseriformes (aves canoras), las que si bien sólo están representadas por 9 especies, el valor de Rtm de 5,6 se debe a que éstas se reparten en 7 géneros y cuatro familias diferentes. Esto además se asocia con atributos ecológicos diferentes, como es el uso de hábitats. Por ejemplo, la familia Furnariidae, que incluye al género Cinclodes o churretes, son especies que frecuentan las riberas de los ríos, mientras que las familias Tyrannidae (e.g. Tachuris rubrigastra o siete colores), Trogloditidae (Cistothorus platensis o chercán de las vegas), e Icteridae (Chrysomus thilius o trile), albergan especies que habitan pajonales densos, en vegas y cuerpos lénticos poco profundos.

De acuerdo al grado de persistencia de las aves acuáticas de Chile, 91 son consideradas residentes, es decir un $69 \%$, mientras que 13 son visitantes regulares, las que en su mayoría arriban anualmente al país durante los meses de primavera y verano. Un número considerable de especies corresponden sólo a registros esporádicos (28), en su mayoría localizados en el extremo norte del país, como es el caso de aves cuya distribución es mas bien tropical o subtropical (e.g. Egretta tricolor, E. caerulaea, y Porphyrio martinicus). Esto sugiere que el extremo norte de nuestro país constituye un área de distribución marginal para varias especies propias de ambientes más septentrionales. Un caso extremo lo constituye la garza de los arrecifes (E. sacra), para la cual existe un solo registro en Arica y cuyo rango distribucional se sitúa predominantemente en el sudeste asiático y Oceanía.

Además de ser el orden de aves acuáticas más diverso, el que incluye el mayor número de especies migratorias es el de los caradriformes (Anexo I). Aquí se encuentran los playeros y chorlitos, los cuales preferentemente habitan zonas costeras arenosas o pedregosas en busca de áreas de descanso y alimentación. Algunas de estas especies arriban desde el hemisferio norte y permanecen en Chile durante el período estival, aunque no se reproducen en las costas chilenas, como es el caso de Calidris alba. $\mathrm{Al}$ respecto, es conocida internacionalmente la importancia de las costas chilenas por el gran número de aves migratorias de larga distancia que las visitan. Estos hábitats ofrecen áreas de descanso y permanencia durante la época no reproductiva, concentrando miles de chorlos y playeros provenientes del Hemisferio Norte (Sallaberry et al. 1996). Entre estas áreas cuentan la Bahía de Mejillones (II Región) y la Bahía de Coquimbo (IV Región) en el norte. Para esta última zona, Tabilo et al. (1996) destacan que las bahías de Coquimbo y Tongoy presentan una alta variedad de microambientes acuáticos. Esta podría ser la razón de por qué albergan una riqueza de aves tan alta. Varios humedales costeros de Chile central son reconocidos además como importantes sitios de anidación, no solo para aves migratorias, sino también para especies residentes. Algunos de ellos están protegidos como Reservas Naturales, como es el caso de Laguna Torca $\left(34^{\circ} 10^{\prime} \mathrm{S}, 71^{\circ} 38^{\prime} \mathrm{W}\right)$, Lago Peñuelas $\left(33^{\circ} 10^{\prime} \mathrm{S}, 71^{\circ} 38^{\prime} \mathrm{W}\right)$ y Laguna El Peral $\left(33^{\circ} 30^{\prime} \mathrm{S}, 71^{\circ} 35^{\prime} \mathrm{W}\right)$ (Vilina \& López-Calleja 1996). Sin embargo, es en la V Región donde se ubica el más importante humedal costero de Chile central. El humedal "Estero El Yali", por su riqueza específica, diversidad y abundancia de aves acuáticas. 


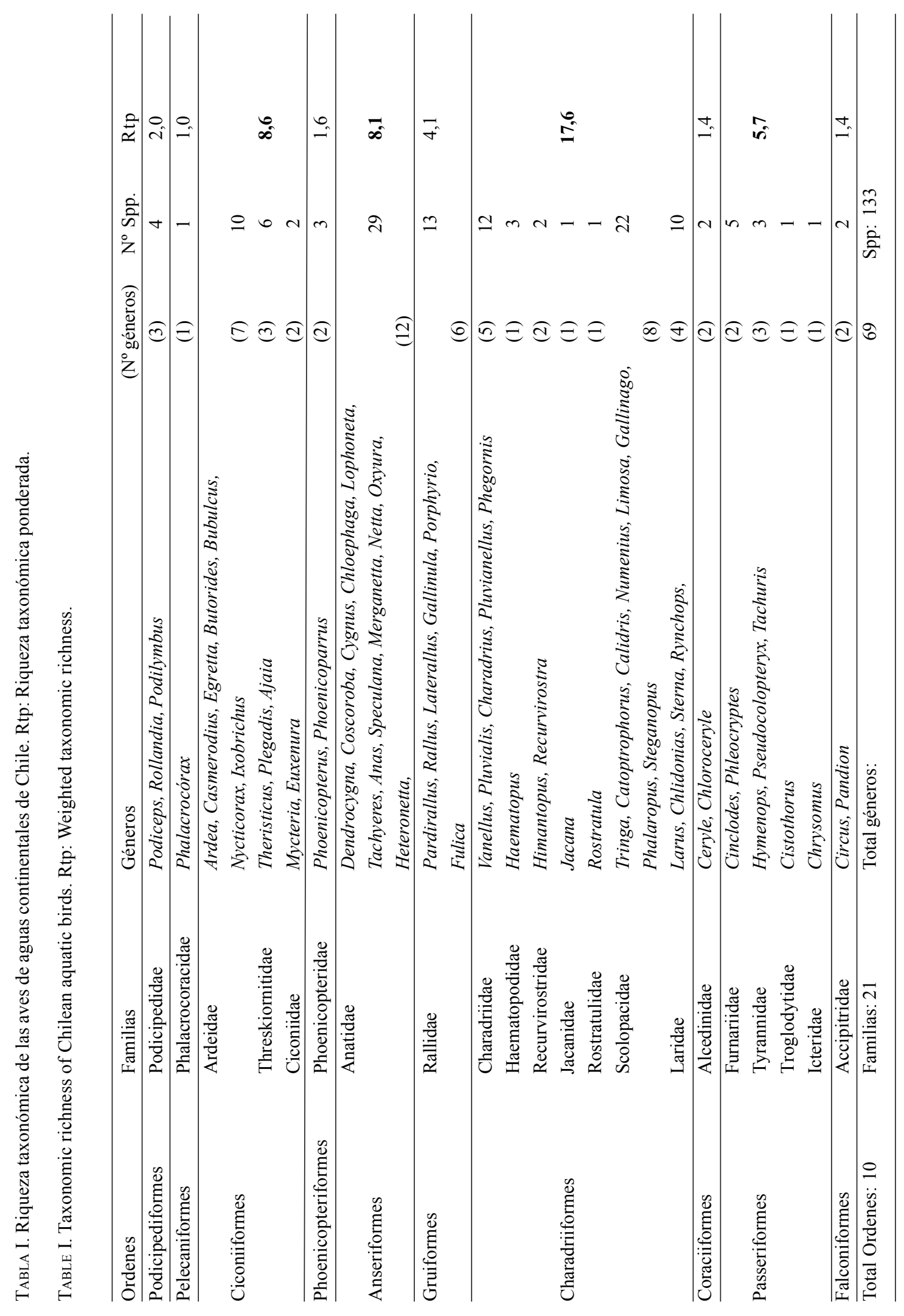


En el centro-sur de Chile hay que destacar también la zona de playas de Rocuant en Talcahuano, VIII Región, sitio al que arriban todos los años, entre otras especies, abundantes grupos de Calidris alba provenientes de Norteamérica (Klesse 1990). Más al sur, en la Región X, dos sitios también son importantes para especies migratorias. Uno es el Seno de Reloncaví en Puerto Montt (Sallaberry \& Tabilo 1990), y otro es el Santuario de la Naturaleza Carlos Andwanther en el río Cruces. Este ultimo es relevante como sitio de residencia y para grupos migratorios de la especie Cygnus melanocoryphus, especie altamente dependiente de humedales con abundante vegetación sumergida (Schlatter 1998).

En cuanto al tipo de hábitat general que usan las aves acuáticas de Chile, del total de especies, un $64 \%$ habitan exclusivamente en ambientes francamente interiores, mientras que un $15 \%$ usa sólo los ambientes transicionales ente el mar y la desembocadura de los ríos. Entre estos últimos las especies predominantes son los caradriformes de las familias Scolopacidae y Laridae. El porcentaje restante está constituido por especies generalistas que habitan indistintamente en ambos tipos de ambientes (21\%). Entre éstos cabe mencionar por ejemplo varias especies de chorlos del género Charadrius, y especies de los géneros Tringa (pitotoyes) y Larus (gaviotas). Los ambientes ecotonales son áreas que concentran una alta diversidad, dado que reúnen especies que predominan en los hábitats que se sobreponen. Por ende, albergan una mayor riqueza específica por unidad de área. Por ejemplo, en un estudio realizado en el estuario de Schelde en la frontera de Holanda y Bélgica, a lo largo de un gradiente de salinidad, se pudo detectar este patrón (Ysebaert et al. 2000). Esta tendencia parece cumplirse también para algunos estuarios chilenos como el de Tubul en la VIII Región (Carrasco 2003), lo que sugiere que dichos ambientes constituyen áreas valiosas para la conservación de un gran número de especies en áreas relativamente reducidas. En general, la riqueza de la avifauna acuática es alta si se tiene en mente la superficie disponible en relación a otros ambientes como por ejemplo el boscoso. Esta diversidad relativamente alta de aves acuáticas se asocia, por una parte, a la alta heterogeneidad y oferta de hábitats de los humedales a escala local. Por otra parte, esta alta diversidad se relaciona con la diversidad bioclimática de nuestro país, que permite la existencia de una gran variedad de tipos de ambientes hú- medos, como por ejemplo, salares en los desiertos, tundra subantártica, turberas, estuarios, ríos salados, lagos oligotróficos, bofedales y fiordos con aguas menos salobres en superficie (Scott \& Carbonell 1986).

\section{ZOOGEOGRAFÍA}

La asociación avifaunística de las ecorregiones chilenas mostró un arreglo consistente con la ubicación geográfica de cada área (Fig. 1). La tendencia es que la mayor diferenciación es para las ecorregiones del norte y la Cordillera de los Andes. Sin embargo, esta última no presenta especies propias, constituyendo un área que incorpora aves que habitan también zonas más bajas a lo largo de todo el país. La ecorregión que incluye un mayor número de especies exclusivas es la Desértica, con 12 taxa correspondientes al 9,1\% del total del país, donde una proporción importante de estos endemismos corresponden a aves playeras (Anexo I). La segunda ecorregión con más especies no compartidas es la Tropical, con 10 especies y un 7,6\% del total de Chile. Un número importante de tales especies son de distribución local restringida, como por ejemplo las parinas $(P h$. andinus y $P h$. jamesi) o algunas cuyo límite distribucional sur es el altiplano chileno - argentino, pero su distribución principal es más al norte. De hecho, la mayor concentración de registros esporádicos para Chile ocurre en el extremo norte del país, lo que se puede interpretar como una zona marginal para la avifauna acuática, y de contacto con unidades biogeográficas diferentes. De las restantes ecorregiones, la Mediterránea y la Oceánica comparten entre sí una mayor proporción de especies y por ende aparecen con un mayor valor de índice de similitud. Sus endemismos son del $3 \%$ y $1,5 \%$ respectivamente. Finalmente la ecorregión Oceánica transandina chilena presenta dos especies no compartidas (Pluvianellus socialis y Chloephaga rubidiceps), con un $1,5 \%$ de endemismos respecto al total chileno. Las tres ecorregiones con mayor riqueza de especies son la Desértica (94 spp), Mediterránea (92 spp) y Oceánica ( 88 spp). Nuestros resultados concuerdan en términos generales con el análisis de endemismos para aves acuáticas de Sudamérica realizado por Fjeldsa (1985). El destaca dos centros de endemismos, uno concentrado en el altiplano y desierto chileno-peruano, concordante con la ecorregión Tropical de este trabajo, y otro ubicado 


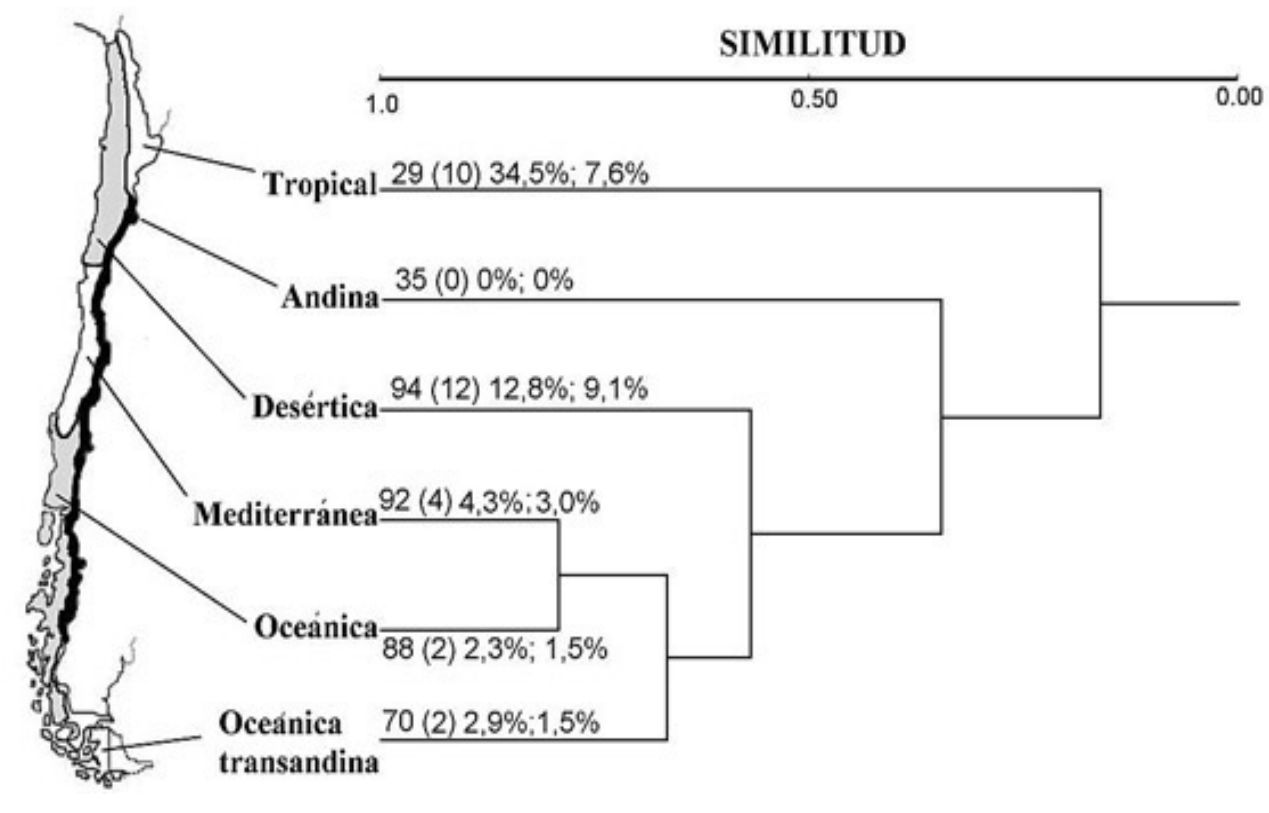

Figura 1. Dendrograma que muestra las similitudes de composición específica de aves acuáticas (Similitud de Jaccard), para las principales ecorregiones chilenas. Los números sobre las ramas indican respectivamente: la riqueza de especies de cada ecorregión, el número de especies exclusivas (en paréntesis), el porcentaje de especies exclusivas respecto al total de cada ecorregión y porcentaje de exclusivas respecto al total registrado para Chile.

FIGURE 1. Dendrogram showing similarities based in the specific composition of aquatic birds (Jaccard Index), for the general Chilean ecoregions. Numbers above the nodes show: species richness for each ecoregion, number of private species (in parenthesis), percentage of private species in respect to the total of each ecoregion, and percentage of private species in respect to the total for Chile.

en el sur de Sudamérica, correspondiente a las regiones Mediterránea, Oceánica y Oceánica Trasandina.

\section{CONSERVACIÓN DE AVES ACUÁTICAS Y HUMEDALES}

Una especie de ave acuática es considerada extinta tanto para el país como para todo su rango distribucional (Numenius borealis, zarapito boreal). Dicha ave migratoria abundaba a principios de siglo conformando numerosas bandadas en ambas américas, y debido a la intensiva caza todas sus poblaciones desaparecieron. Otras veinticinco especies que habitan humedales en Chile están catalogadas en alguna categoría de conservación (Tabla II). De acuerdo al Libro Rojo de los Vertebrados de Chile (Glade 1989), 4 especies están en la categoría En Peligro. Otras 10 especies son Vulnerables, 6 son Raras y 5 Inadecuadamente Conocidas. Den- tro de estas últimas llama la atención esta clasificación para la especie Rallus antarcticus (pidén austral), especie considerada extinta para el centro del país. La lista de la IUCN la ubica en la categoría de Vulnerable, la cual parece más adecuada si se tiene en cuenta además los escasos registros de las ultimas cinco décadas. Hasta 1959 existían registros para Buenos Aires, rió Negro, Chubut, Santa Cruz en Argentina, y para Chile en Tierra del Fuego, Valparaíso, Santiago, Colchagua, Llanquihue y Magallanes. Después de ese año, no hubo registro alguno hasta 1990 (tres sitios). R. antarcticus fue redescubierto en 1998, y ha seguido avistándose en al menos seis localidades, tanto en la provincia de Santa cruz, Argentina, como en Magallanes, Chile, donde la población local más abundante es de aproximadamente 35 individuos ${ }^{3}$. Entre las causas

${ }^{3}$ http://www.birdlife.org/datazone/search/species_search. html action $=$ SpcHTMDetails . asp \& sid $=2869$ \& $\mathrm{m}=0$ 
Aves de aguas continentales de Chile: Victoriano, P. et AL.

TABLA II. Lista de especies de aves acuáticas chilenas clasificadas en categorías de conservación. (*): Especie actualmente considerada extinta en Chile central según el criterio de antigüedad de último registro. NT: "Near Threatened", susceptible de ser amenazada; VU: "Vulnerable". IC: Inadecuadamente conocida; V: Vulnerable; FP: Fuera de peligro. II: Incluida en el Apéndice II.

TABLE II. List of Chilean species of aquatic birds classified in conservation categories. (*) Species extinct in Central Chile according the last register. NT: Near Threatened, VU: Vulnerable; IC: Insufficient information; V:Vulnerable; FP: "Fuera de Peligro"; II: Included in Appendix II.

\begin{tabular}{|c|c|c|c|c|}
\hline Especies/ Categorías (Glade, 1989) & Nombre común & IUCN & CONAMA(VIII Región) & CITES \\
\hline \multicolumn{5}{|l|}{ EN PELIGRO } \\
\hline 1.Rostratula semicollaris & Becacina pintada & & $\mathrm{IC}$ & \\
\hline 2.Chloephaga rubidiceps & Canquén colorado & & - & \\
\hline 3. Coscoroba coscoroba & Cisne coscoroba & & - & II \\
\hline 4.Plegadis chihi & Cuervo de pantano & & $\mathrm{IC}$ & \\
\hline \multicolumn{5}{|l|}{ VULNERABLES } \\
\hline 1.Theristicus melanopis & Bandurria & & $\mathrm{IC}$ & \\
\hline 2. Gallinago paraguaiae & Becacina & & - & \\
\hline 3.Cygnus melanocoryphus & Cisne cuello negro & & $\mathrm{V}$ & II \\
\hline 4.Plegadis ridgwayi & Cuervo de la puna & & - & \\
\hline 5.Phoenicopterus chilensis & Flamenco chileno & NT & $\mathrm{IC}$ & II \\
\hline 6.Phoenicoparrus jamesi & Parina chica & NT & - & II \\
\hline 7.Phoenicoparrus andinus & Parina grande & VU & - & II \\
\hline 8.Chloephaga melanoptera & Piuquén & & - & \\
\hline 9.Fulica cornuta & Tagua cornuda & NT & - & \\
\hline 10.Fulica gigantea & Tagua gigante & & - & \\
\hline \multicolumn{5}{|l|}{ RARAS } \\
\hline 1.Pluvianellus socialis & Chorlo de Magallanes & NT & - & \\
\hline 2.Ardea cocoi & Garza cuca & & $\mathrm{IC}$ & \\
\hline 3.Larus serranus & Gaviota andina & & - & \\
\hline 4.Ixobrychus involucris & Huairavillo & & $\mathrm{V}$ & \\
\hline 5.Anas bahamensis & Pato gargantillo & & $\mathrm{IC}$ & \\
\hline 6.Heteronetta atricapilla & Pato rinconero & NT & $\mathrm{IC}$ & \\
\hline \multicolumn{5}{|c|}{ INADECUADAMENTE CONOCIDAS } \\
\hline 1.Tachyeres patachonicus & Pato quetru volador & & - & \\
\hline 2.Anas platalea & Pato cuchara & & FP & \\
\hline 3.Laterallus jamaicensis & Pidencito & NT & $\mathrm{IC}$ & \\
\hline 4. Rallus antarcticus* & Pidén austral $(*)$ & VU & - & \\
\hline 5.Pseudocolopteryx flaviventris & Pájaro amarillo & & $\mathrm{IC}$ & \\
\hline
\end{tabular}

de su disminución numérica, la IUCN señala la perdida de hábitat y el incremento de la superficie destinada a agricultura.

Otros tres sistemas de clasificación considerados en este trabajo sugieren diferencias importantes entre sí. En un análisis mediante el uso de índices numéricos de clasificación, CONAMA (1999) reclasifica las aves acuáticas de la Región del Bío-Bío. En él no aparecen especies en categoría de En Peligro, y cambia a la categoría de Inadecuadamente Conocida a $R$. semicollaris (becacina pintada) y Plegadis chihi (cuervo de pantano). Una especie que debiera ser incorporada a la lista de taxa para la Región del BíoBío es C. coscoroba, clasificada como En Peligro para el país por Glade (1989), y que ha sido observa- da en grupos importantes recientemente en humedales costeros de Concepción. La lista de la IUCN, sólo incluye a 8 de las especies presentes en Chile, en su mayor parte en la categoría de NT ("Near Threatened", susceptib les de ser amenazadas).

No existen especies de aves acuáticas endémicas para Chile. Sin embargo, su riqueza y singularidad ecológicas son atributos importantes al momento de considerar la conservación de espacios acuáticos continentales. En el caso específico de los sistemas acuáticos, clasificados como "humedales", éstos están desapareciendo rápidamente. Los humedales representan zonas con límites concretos y que albergan una gran diversidad de fauna y flora, y han sido catalogados como zonas de alta productividad, 
ya que proveen un gran número de recursos para la vida silvestre, representan para muchas formas de vida un espacio para la reproducción y mantienen los sistemas hidrológicos naturales, constituyéndose en zonas ecológicas especiales (Kusler et al. 1994; Muñoz \& Möller 1997; Barbier et al. 1997). La tendencia de los estudios en ecología de aves chilenas de humedal, realizados con fines de conservación, es a abordar aspectos de estructura comunitaria y censos. Hasta hoy, el instrumento de mayor cobertura geográfica en la estimación de abundancias es el Censo Neotropical de Aves Acuáticas, el cual está concebido como una herramienta para determinar la distribución de las aves y monitorear las tendencias de las poblaciones a largo plazo (e.g. Schlatter 1993; Espinosa 2000). Una revisión acuciosa realizada por Schlatter et al. (en prensa) incluye 282 si- tios, entregando los valores biológicos, beneficios y amenazas de cada uno de estos humedales. No obstante, según estos autores, Chile podría superar un inventario de 2000 sitios de humedales, tanto naturales como artificiales. En relación a la proporción de humedales existentes en cada región, existen cuatro regiones que han sido proporcionalmente más estudiadas que las demás, donde el porcentaje de estudios ornitológicos es mayor al porcentaje de humedales existentes (Fig. 2). Estas son la IV, V, VII y R.M. La mayor parte de los estudios sobre aves acuáticas han sido desarrollados en las regiones $\mathrm{V}$ y X, donde destacan sitios como el Estero El Yali y los humedales del río Cruces, lo cual se ha debido probablemente a la presencia en estas regiones de los centros de estudio ornitológico más importantes del país.

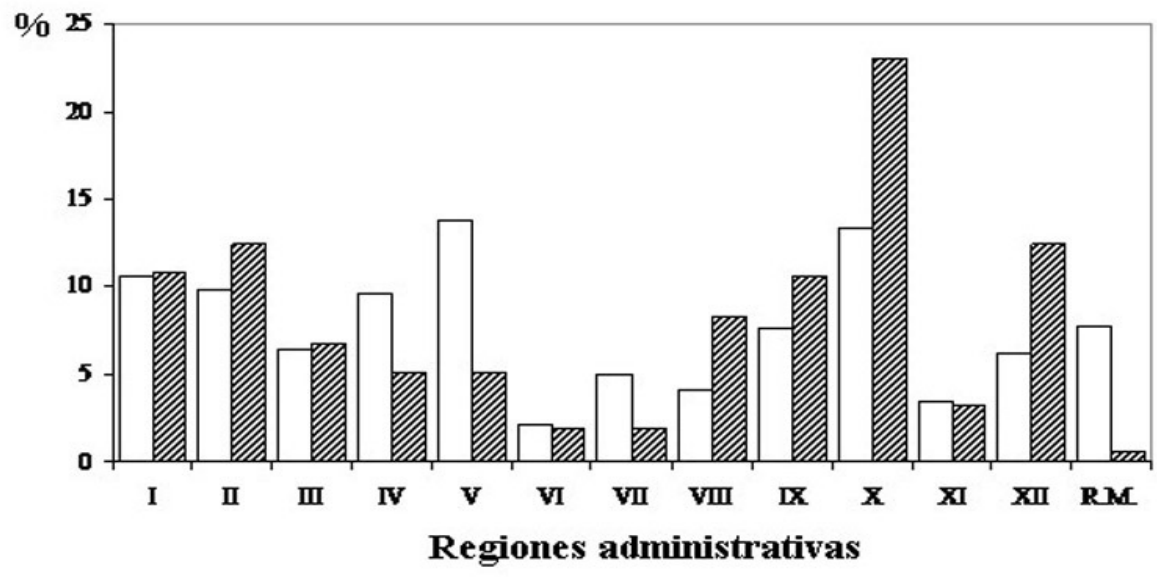

FiguRa 2. Gráfico para el porcentaje de estudios de aves acuáticas (blanco) y porcentaje de humedales (achurado), (Scott \& Carbonell, 1986) por Región administrativa de Chile.

FIGURE 2. Graphic for the percentage of studies on aquatic birds (white), and percentage of wetlands (hatched) (Scott \& Carbonell, 1986), for each Chilean administrative Region.

Sin embargo, a pesar de que la X Región concentra cerca de un $13 \%$ de los estudios, ésta se encuentra sub representada en la literatura ornitológica nacional, si se considera que concentra más del $23 \%$ de los humedales del país.

Junto a la Región de Los Lagos (X), otras cuatro regiones muestran un porcentaje menor de estudios de aves que de humedales (II, VIII, IX y XII). Respecto a los tipos de humedales continentales que existen en Chile, los más estudiados son los estuarinos. Los sistemas lacustres y ribereños son medianamente abordados, mientras que los palustres y artificiales han sido muy poco estudiados (Muñoz \& Möller 1997). Dentro de éstos, los más investigados son aquellos definidos como áreas de protección Ramsar, las que suman en total ocho para Chile y se encuentran distribuidas entre la I y $\mathrm{X}$ regiones (Santuario de la Naturaleza Laguna Conchalí, IV Región; Santuario de la Naturaleza Carlos Andwanther, X Región; Humedal El Yali, V 
Aves de aguas continentales de Chile: Victoriano, P. et aL.

Región; Complejo Laguna del Negro Francisco Laguna Santa Rosa, III Región; Salar de Surire, Salar de Tara y Salar de Huasco, los tres en la I Región, y el Sistema Hidrológico de Soncor en la II Región).

Naranjo (1995) calculó que en 1986 el Sistema Nacional de Areas Silvestres Protegidas del Estado protegía cerca del 40,3\% de sus humedales. Estos ambientes acuáticos se encuentran mejor representados, dentro de este sistema, en la región austral, es decir, en las regiones bioclimáticas correspondientes al tipo oceánico subantártico y oceánico templado-frío, mientras que se encuentran mal representadas en las regiones bioclimáticas mediterránea y en el sector costero (Scott \& Carbonell 1986). Los inventarios de humedales en Chile comenzaron en 1984, cuando Schlatter \& Espinosa publicaron una compilación realizada por Scott \& Carbonell (1986) para el Inventario de Humedales de la Región Neotropical. En esa revisión se propusieron 50 sitios para nuestro país, abarcando una superficie de 5.500 .500 há, centrándose preferentemente en el Sistema Nacional de Areas Silvestres Protegidas del Estado (SNASPE), registrándose un total de $22,4 \%$ de sitios para la X Región, un $12,1 \%$ para la II y XII Región, la VI y VII con tan sólo un $1,7 \%$ y mientras que para la VIII Región un $8,6 \%$.

La cantidad de humedales incluidos en los artículos que fueron analizados es muy baja en comparación a la existente, la zona que presenta mayor representatividad con respecto a las áreas registradas, es la II Región con 15 humedales, seguida de la I Región con 9, lo que al parecer alcanzaría al $100 \%$, mientras que en la VII y XI Región no se registró ningún estudio en la revisión. Al comparar con los humedales considerados por los Censos Neotropicales de Aves Acuáticas, la situación cambia. La V Región es la que presenta una mayor cantidad de sitios muestreados, con un promedio de 18 lugares, presentando un máximo de 20 y un mínimo de 9 , lo que representa un $95 \%$ y un $43 \%$ del total, respectivamente. Le sigue la X Región, con un promedio de 33 áreas, teniendo un máximo de 46 y un mínimo de 14 humedales, representando el $57 \%$ y $17 \%$ del total. Por otro lado, las regiones con menor representatividad de sitios en estos censos son la III, VI y VIII Región, con sólo un sitio como promedio, lo que equivale al $6,6 \%$, $6,2 \%$ y $5,9 \%$ respectivamente

\section{PRINCIPALES COLECCIONES Y CENTROS DE ESTUDiO}

Actualmente en Chile existen ocho centros importantes dedicados al estudio de las aves; 1) La Universidad de Antofagasta (II Región), cuya área principal de interés es la ecofisiología de aves costeras; 2) la Universidad de Valparaíso (V Región), dedicada principalmente al estudio de la bioacústica y etología; 3) la Universidad de Chile (Región Metropolitana), con investigaciones en la biología de la migración interhemisférica, especialmente chorlos y playeros, autoecología de aves antárticas, relaciones ave-planta, biología de la conservación y biología del desarrollo; 4) la Pontificia Universidad Católica de Chile (Región Metropolitana) con investigaciones en el campo de la ecología de comunidades de aves terrestres y antárticas, con énfasis en algunos aspectos de conservación; 5) el Museo Nacional de Historia Natural (Región Metropolitana) con investigaciones museológicas y listados de distribución de aves; 6) la Universidad Austral (X Región), con investigaciones principalmente en el campo de las aves de humedales, esencialmente su biología poblacional; 7) la Universidad de Magallanes (XII Región), con trabajos sobre la distribución de especies, y recientemente en la Universidad de Concepción (VIII Región), principalmente dedicado a estudiar la avifauna de humedales interurbanos de esa zona. Además debe considerarse la Corporación Nacional Forestal (CONAF), que desarrolla diversos programas de seguimiento, principalmente en especies clasificadas en categorías de conservación, y la Unión de Ornitólogos de Chile, que desarrolla una importante labor en el conocimiento de la avifauna chilena (Lazo \& Silva 1993).

Dos sitios de la red incluyen un listado de ornitólogos chilenos, entre los que algunos de ellos se dedican al estudio de aves acuáticas continentales $^{4}$. Una lista con sus direcciones se incluye en el Anexo II. Las principales colecciones museológicas que incluyen aves acuáticas se encuentran en el Departamento de Zoología de la Universidad de Concepción, en el Instituto de Zoología de la Universidad Austral de Chile, en la Universidad de Magallanes y en el Museo Nacional de Historia Natural de Santiago.

\footnotetext{
${ }^{4} \mathrm{http}: / /$ www.avesdechile.cl/contacto.htm

${ }^{5} \mathrm{http} / / /$ www.geocities.com/RainForest/4372/DIRECTO.HTM
} 


\section{ESTADO DE CONOCIMIENTO Y PROYECCIONES FUTURAS}

Una visión general del estado de conocimiento sobre las aves de Chile puede obtenerse a partir de la exhaustiva recopilación de literatura de Enrique Sil$\mathrm{va}^{6}$. En esta lista se incluyen las publicaciones ornitológicas desde 1847 al 2005. Según Lazo \& Silva (1993), de los trabajos de investigación ornitológica publicados durante el periodo 19701992 , el $28 \%$ se refirió a estudios realizados en distribución de especies, un $14 \%$ a interacciones interespecíficas, un $6 \%$ a censos y/o abundancia y sólo el $4 \%$ dedicado a protección y conservación de especies. Schlatter (1979) también realizó un análisis de las tendencias de la ornitología chilena, en el cual concluyó que las áreas de mayor desarrollo estaban dedicadas a estudios distribucionales seguidos de censos y dinámica poblacional, mientras que los referidos a protección y conservación son menos numerosos. La taxonomía de aves acuáticas a nivel de especies parece haberse estabilizado. Sin embargo, son pocas las especies presentes en Chile que son incluidas en análisis de relaciones filogenéticas, y son menos aún los trabajos basados en estudios moleculares. A escala de filogenia profunda el clásico de Sibley et al. (1988) mostró un arreglo para las familias de aves basado en hibridización de ADN. Recientemente Fain \& Houde (2004) y Poe $\&$ Chubb (2004) reanalizaron gran parte de los grandes clados mediante secuencias de ADN, incluyendo grupos de aves acuáticas. Como parte del megaproyecto en desarrollo "Tree of Life (AToL)", financiado por el gobierno de los Estados Unidos, está la idea de reconstruir la filogenia de los principales grupos de aves del mundo (Bennu, 2004).

La mayor parte del conocimiento filogenético de especies acuáticas presentes en Chile ha surgido de estudios hechos en otros países. Esta es una de las ventajas de la amplia distribución geográfica de algunos taxa. Un ejemplo de esto es el trabajo de Livezey (1986) sobre las relaciones de parentesco al interior del genero Tachyeres (patos vapor), y de los Anatidae en general (Livezey 1996). Otro ejemplo un tanto más extensivo, es el de Donne-Gousse et al. (2002) quienes estudiaron la filogenia de todo el orden Anseriformes mediante ADN mitocondrial, y donde incluyen algunas

\footnotetext{
${ }^{6} \mathrm{http}: / /$ www.bio.puc.cl/auco/artic01/ornito01.htm
}

especies presentes en Chile. Algo similar es para Gruiformes y Pelecaniformes (Houde et al. 1997; Siegel-Causey 1997). Para las taguas y pidenes (Gruiformes, Rallidae) está el análisis filogenético de la familia hecho por Livezey (1998), aunque no incluye especies presentes en Chile. En otro caso, Van Tuinen et al. (2001) estudiaron los procesos de convergencia y divergencia evolutiva en aves acuáticas, e incluyó secuencias de las especies Charadrius semipalmatus, Phalacrocórax brasilianus y Nycticorax nycticorax. Recientemente se encontró evidencia molecular y morfológica de que el probable grupo hermano de los flamencos es el orden Podicipediformes, donde están los pimpollos y las hualas (Mayr 2004). En este último trabajo se incluyen, además de Phoenicopterus chilensis, las especies Dendrocygna viduata, Oxyura jamaicensis y Nycticorax nycticorax. McCracken et al. (1999) estudiaron los patrones de evolución de algunos caracteres morfológicos, en patos, apoyados en evidencia molecular, en cuyo análisis incluyen las dos especies de Oxyura presentes en Chile, y a Cygnus melanocoryphus como grupo externo. Existen más publicaciones sobre la filogenia de otros grupos de aves acuáticas, como por ejemplo para caradriformes (Joseph et al. 1999; Crochet \& Desmarais 2000), pero no es la finalidad de este trabajo entregar una descripción exhaustiva de esta literatura. Es relevante destacar que gran parte de los estudios evolutivos por sobre el nivel de especie han surgido de instituciones de otras partes del mundo, debido a la amplia distribución geográfica de muchas aves acuáticas que existen en Chile. Sin embargo, en otros casos es pertinente un análisis más restringido geográficamente, como por ejemplo para probables grupos naturales que se distribuyan sólo en la parte sur de Sudamérica. Este puede ser el caso de especies poco vágiles, como las taguas del sur de Sudamérica, u otras especies de rállidos.

A una escala evolutiva menor, resulta de fundamental importancia para fines de conservación de variantes intraespecíficas el determinar los niveles de divergencia evolutiva al interior de especies de amplia distribución. A diferencia de las investigaciones filogenéticas a nivel de especies o de jerarquías mayores, los análisis filogeográficos deben ser necesariamente realizados en el país. Esto permitiría, entre otros aspectos, definir unidades 
evolutivas significativas (ESUs), considerando atributos de singularidad y diversidad genéticas (Crandall et al. 2000). De todas las aves de aguas continentales presentes en Chile, para 17 especies se han descrito 35 razas geográficas, de las cuales la mayoría se concentran en los extremos norte y sur del país (11 en Desértica y Tropical, y 7 en la Oceánica y Oceánica transandina). Algunos ejemplos son Podiceps occipitalis (blanquillo), Pardirallus sanguinolentus (pidén), Tachuris rubrigastra (siete colores) y Merganetta armata (pato cortacorrientes). Esto sugiere un rol importante del aislamiento relativo de algunas poblaciones, y de probables adaptaciones locales o eventos históricos que sería interesante aclarar por medio de herramientas moleculares. Por lo anterior resulta fundamental realizar análisis filogeográficos para esas especies de aves, con el fin de establecer unidades de conservación subespecíficas basadas en información microevolutiva.

En un ámbito complementario al anterior, es necesario incrementar el grado de conocimiento sobre la biología y factores ecológicos relacionados con especies en categorías de conservación. Matus et al. (2000) estudiaron los sitios de reproducción y distribución espacial del canquén colorado Chloephaga rubidiceps, en Magallanes. Corti $\&$ Schlatter (2002) analizaron la dieta del cisne de cuello negro (Cygnus melanocoryphus) en el sur de Chile, donde destacan la relevancia de la vegetación acuática para la persistencia de sus poblaciones. Schlatter (1998) hizo una buena síntesis acerca de la biología y fluctuaciones poblacionales de C. melanocoryphus en el rió Cruces, donde deja de manifiesto la relevancia de este santuario para la conservación de la población más importante de cisne de cuello negro en Chile. Brewer \& Vilina (2002) publicaron valiosos antecedentes sobre el comportamiento reproductivo y de crianza del cisne coscoroba (Coscoroba coscoroba) en Chile central. Mascitti \& Bonaventura (2002) estudiaron las variaciones de abundancia y patrones de uso del hábitat de flamencos altiplánicos. Estos son ejemplos de aspectos relevantes de la biología de las especies que, en conjunto con antecedentes sobre los factores ambientales que afectan las abundancias poblacionales, serán de gran ayuda en acciones futuras de conservación. Ejemplo de esto ultimo es el trabajo de Cornelius et al. (2001), quienes relacionaron la presencia humana con los patrones de estructura comunitaria de aves costeras en Chile central. Gonzalez \& Victoriano (2005) destacan la fragilidad de los humedales de la zona de Concepción y el rol de la estructura del hábitat en la conservación de la avifauna. En algunos casos son los cambios naturales y a gran escala los que pueden afectar las abundancias de aves acuáticas, como es el caso del fenómeno del Niño y su efecto sobre las abundancias del cisne de cuello negro en El Yali, en Chile central (Vilina et al. 2002). Son muy pocas las publicaciones que explícitamente tratan las medidas de conservación de aves acuáticas chilenas. Excepciones las constituyen los trabajos de Myers et al. (1987) para aves playeras migratorias, Parada (1990) para flamencos en el norte de Chile, y Rottmann \& López-Calleja (1992) para aves chilenas en general.

Por último, si bien se recopilan numerosos datos sobre las abundancias de una amplia gama de especies de humedales mediante el Censo Neotropical de Aves Acuáticas, y con una cobertura geográfica importante, se hace necesario un análisis integral de tal información. Con esto podrían sacarse valiosas conclusiones, tanto acerca de las tendencias poblacionales a gran escala espacio temporal, como sobre patrones macroecológicos. Todos estos aspectos podrían además contribuir a implementar métodos más objetivos (índices) de clasificación para determinar estados de conservación de las aves acuáticas chilenas, lo que a su vez optimizará las acciones de conservación frente a futuros proyectos de desarrollo en el país.

\section{AGRADECIMIENTOS}

Este trabajo es parte del proyecto Instrumental Científico-2001: "Implementación de un grupo de estudios de biodiversidad regional: una base para el ordenamiento territorial" de la Dirección de Investigación. Universidad de Concepción. También es parte de las actividades del Grupo de Investigación 03.K3.01 "Biodiversidad y Conservación de Ecosistemas Terrestres". Parte de la información fue recopilada en el marco del proyecto 205.113.0671sp. Agradecemos a la comisión organizadora del XI Taller Nacional de Limnología de Chile por promover la publicación de este trabajo. 


\section{BIBLIOGRAFIA}

Araya, B.M., M. Bernal, R. Schlatter \& M. Sallaberry. 1995. Guía de campo de las aves de Chile. 4ta ed. Ed. Universitaria - Santiago. 405p.

Araya, B.M. \& G. Millie. 1996. Guía de campo de las aves de Chile. 4ta ed. Ed. Universitaria - Santiago. $405 \mathrm{p}$

Avise, J. 2004. Molecular Markers, Natural History, and Evolution. Segunda Edición. Chapter 1. Sinauer. Massachusetts. 684 pags.

Barbier, E., M. Acreman \& D. Knowler. 1997. Valoración Económica de los Humedales - Guía para Decisores y Planificadores. Oficina de la Convención Ramsar, Gland, Suiza. 143 pp.

Bennu, D. A. 2004. The Evolution of Birds: An Overview of the Avian Tree of Life. Lab Animal 33(5): $42-$ 48

Brewer, G. \& Y. ViLina. 2002. Parental Care behaviour and double-brooding in Coscoroba swan in Central Chile. Waterbirds. 25: 278-284.

Canevari, P., G. Castro, M. Sallaberry \& L.G. NaranJo. 2002. Guía de identificación de aves Playeras en el Geotrópico. Impresora FERIVA S.A. Cali Colombia. 141p.

CARrasco, P. 2003. Variaciones de la estructura de la avifauna a lo largo de un gradiente de salinidad en el humedal Tubul-Raqui, VIII Región, Chile. Unidad de Investigación. Universidad de Concepción. Mecanografiada. 97 págs.

CONAMA, 1999. Evaluación del estado de Conservación de las Aves de Humedal de la Región del Bío-Bío. 38 pp.

Cornelius, C., S. A. Navarrete \& P. A. Marquet. 2001. Effects of Human Activity on the Structure of Coastal Marine Bird Assemblages in Central Chile. Conservation Biology 15(5):1396-1404.

Corti, P. \& R. Schlatter. 2000. Feeding ecology of the Black-necked swan Cygnus melancoryphus (Molina, 1782) in two wetlands of Southern Chile. Studies on Neotropical Fauna and Environment. 37 : 9- 14.

Crandall, K. O Bininda-Emonds, G Mace \& R Wayne. 2000. Considering evolutionary processes in conservation biology. Trends in Ecology and Evolution 7: 290-295.

Crochet, P. A. \& E. Desmarais. 2000. Slow rate of evolution in the mitochondrial control region of gulls (Aves: Laridae). Molecular Biology and Evolution, 17:1797-1806.

De la Peña, M. \& M. Rumboll. 1998. Birds of Southern South America and Antarctica. Princeton Univ. Press. USA. 304 pags.

Di CAstri F. 1968. Esquisse écologique du Chili. En Biologie de 1'Amérique Australe. CNRS. Paris.

Donne-Gousse, C., V. Laudet, \& C.Hanni. 2002. A molecular phylogeny of anseriformes based on mitochondrial DNA analysis. Molecular Phylogenetic and Evolution. 23(3):339-356.

Espinosa, L.A. 2000. Censo Neotropical de Aves Acuáti- cas 1999 (sección Chile). Boletín Chileno de Ornitología 7: 39 - 47.

Fain, M. G. \& P. Houde. 2004. Parallel radiations in the primary clades of birds. Evolution: 58 (11) 25582573.

Felizola, J.A. 2004. Phylogenetic diversity and conservation priorities under distinct models of phenotypic evolution. Conservation Biology. 18(3): 698-704.

Figueroa, R., J. Cerda \& C. Tala. 2001. Guía de Aves Dulceacuícolas de Aysen. Servicio Agrícola y Ganadero. Ministerio de Agricultura. 102 págs.

Fueldsa, J. 1985. Origin, evolution, and status of the avifauna of Andean Wetlands. Pp 85-112 en P.A. Buckley, M.S. Foster, E.S. Morton, R.S. Ridgely, and F.G. Buckley (Eds.) Neotropical Ornithology. Ornithological Monographs 36. AOU, Washington.

Glade, A. (Ed.), 1989. Libro Rojo de los Vertebrados Terrestres de Chile. CONAF. Santiago, Chile. 95 pp.

GonzÁlez, A. \& P. Victoriano. 2005. Aves de los humedales costeros del Centro-Sur de Chile. Capítulo 35 En: Smith C., J. Armesto \& C. Valdovinos (Eds.) Biodiversidad y Ecología de la Cordillera de la Costa. Fundación Senda Darwin. (En prensa).

Goodall, J.J., A.W. Johnson, \& R.A. Philippi B. 1946. Las aves de Chile, su conocimiento y sus costumbres. Vol. 1 Buenos Aires: Platt Establecimientos Gráficos S.A. 358pp.

Goodall, J.J., A.W. Johnson, \& R.A. Philippi B. 1951. Las aves de Chile, su conocimiento y sus costumbres. Vol. 2 Buenos Aires: Platt Establecimientos Gráficos S.A. 445pp.

Houde, P., A. Cooper; E. Leslie; A.E. Strand \& G.A. Montano. 1997. Phylogeny and evolution of $12 \mathrm{~S}$ rDNA in Gruiformes (Aves). Chap.5. In: D. P. Mindell (Ed.) 1997. Avian Molecular Evolution and Systematics. $121-154$.

Jaramillo, A. 2003. Birds of Chile. Princeton Field Guides. Princeton. 240 pp.

Johnson, A.W. 1965. Birds of Chile and Adjacent Regions of Argentina, Bolivia and Perú. Vol. 1. Platt Establecimientos Gráficos, Buenos Aires.

Johnson, A.W. 1967. Birds of Chile and Adjacent Regions of Argentina, Bolivia and Perú. Vol. 2 Platt Establecimientos Gráficos, Buenos Aires.

Joseph, L., E. P. Pessa, L. Christidis. 1999. Phylogeny and biogeography in the evolution of migration: shorebirds of the Charadrius complex. Journal of Biogeography, 26:329-342.

Kennedy, M. E. 1977. Requisitos para el hábitat de reproducción de la Tagua común y recomendaciones técnicas de manejo para aumentar esta especie. Medio Ambiente 2 (2):107-116.

KLESSE, M.C. 1990. Ecología trófica y distribución espacial de Calidris alba (Caradriformes) en el sector de Isla Roquant (VIII Región, Chile). Tesis de Grado de Magíster en Ecología. Universidad de Chile. Mecanografiada. 
Aves de aguas continentales de Chile: Victoriano, P. et AL.

Kusler, J., W. Mitsch \& J. Larson. 1994. Humedales. Investigación y Ciencia 210: 6-13.

Lazo, I. \& E. Silva. 1993. Diagnóstico de la ornitología en Chile y recopilación de la literatura científica publicada desde 1970 a 1992. Revista Chilena de Historia Natural 66: 103-118.

Livezey B. C. 1986. Phylogeny and historical biogeography of steamer-ducks (Anatidae: Tachyeres). Systematic Zoology 35: 458-469.

Livezey, B. C. 1996: A phylogenetic classification of waterfowl (Aves: Anseriformes), including selected fossil species. -Annals of Carnegie Museum: vol. 66 (4):457-496.

Livezey, B. C. 1998. A phylogenetic analysis of the Gruiformes (Aves) based on morphological characters, with an emphasis on the rails (Rallidae). Philosophical Transactions of the Royal Society (Ser. B) 353:1-72.

Mccracken, K. G., J. Harshman; D. A. Mcclellan \& A. D. Afton. 1999. Data Set Incongruence and Correlated Character Evolution: An Example of Functional Convergence in the Hind-Limbs of Stifftail Diving Ducks. Systematic Biology. 48(4):683-714.

Martínez, D. \& G. González. 2004. Las Aves de Chile. Nueva Guía de Campo. Ediciones del Naturalista. Santiago de Chile. 620 págs. 181 láminas.

Mascitti, V. \& S. Bonaventura. 2002. Patterns of abundance, distribution and habitat use of flamingos in the high Andes, South American flamingos. Condor 104: 73-83.

Matus, R., O. Blank, D. Blanco, J Madsen, L Venegas \& G Mateáis. 2000. El canquén colorado (Chloephaga rubidiceps): antecedentes sobre sitios de reproducción y concentración en la XII Región de Magallanes, Chile. Boletín Chileno de Ornitología 7: 13-18.

MAYR, G. 2004. Morphological evidence for sister group relationship between flamingos (Aves: Phoenicopteridae) and grebes (Podicipedidae). Zoological Journal of the Linnean Society, 140:157-170.

Molina, J.I. 1782. Saggio sulla Storia Naturale et Civile del Chili. Bologna, 367 pp.

Muñoz, A. \& P. Möller (Eds.) 1997. Conservación de Humedales. Editorial CEA. 460 pags.

Myers, J.P., P.Z. Antas, T. E. Lovejoy, R. I. Morrison, M. Sallaberry, S. E. Senner \& A. Tarak. 1987. Conservation strategy for migratory species: an example with shorebirds (Charadrii). American Scientist 75: 18-26.

Naranjo, L. 1995. An evaluation of the first inventory of south american wetlands. Vegetatio 118 (12): $125-129$.

Philippi, R.A. 1864. Catálogo de las aves chilenas con su distribución geográfica. Investigaciones Zoológicas Chilenas XI: 1-179.

Parada, M. 1990. Flamencos en el norte de Chile, distribución, abundancia y fluctuaciones estacionales del número. Actas I Taller Internacional de Espe- cialistas en Flamencos Sudamericanos. Corporación Nacional Forestal-New York Zoological Society. Antofagasta (Chile) 1: 52-66.

Poe S. \& A. L. Chubb. 2004. Birds in a bush: five genes indicate explosive evolution of avian orders. Evolution, 58(2): 404-415

Ridgely, R. \& G. Tudor. 1989. The Birds of South America. The Oscine Passerines.Univ. Texas Press. Austin. Vol. 1. 516 págs.

Ridgely, R. \& G. Tudor. 1994. The Birds of South America. The Suboscine Passerines. Univ. Texas Press. Austin. Vol. 1. 820 pags.

Riveros G., I. Serey \& P. Drouilly. 1981. Estructura y diversidad de la comunidad de aves acuáticas de la laguna El Peral, Chile Central. Anales del Museo de Historia Natural 14: 189-196.

Rottmann, J. 1995. Guía de Identificación de aves de Ambientes Acuaticos. UNORCH. Santiago de Chile. 80 págs.

Rottmann, J. \& M.V. López-Calleja. 1992. Estrategia Nacional de Conservación de Aves. Serie Técnica 1 (1): 16 pp.

Sallaberry, M. \& E. TABILO. 1990. Importantes áreas de descanso para las aves migratorias en Chile y su conservación. Revista Creces 2 (7): 14-19.

Sallaberry, M., E. Tabilo, C. Klesse \& J. Abarca. 1996. The chilean shorebird network (RECAP). Shorebird Ecology and Conservation in the Western Hemisphere. International Wader Studies 8: 71-78.

Schlatter, R. 1979. Avances de la ornitología en Chile. Archivos de Biología y Medicina Experimental. En Simonetti, J., M. Arroyo, A. Spotorno \& E. Lozada (Eds.). 1995. Diversidad biológica de Chile. CONICYT. Santiago, Chile. 364 pp.

SChlatter, R. 1993. Censo neotropical de aves acuáticas 1991 (sección Chile). Humedales para las Américas. Valdivia, Chile. 26-37.

Schlatter, R. 1998. El cisne de cuello negro (Cygnus melanocoryphus) en Chile. En: Valverde, V. (Ed). La Conservacion de la Fauna Nativa de Chile. CONAF. Santiago de Chile. 179 pags.

Scott, D. \& M. CARbonell (Eds.). 1986. Inventario de Humedales de la Región Neotropical. IWRB. Slimbridge IUCN, Cambridge, United Kingdom. 714 pp.

Sibley, C. G., J. E. Ahlquist, \& B. L. Monroe JR. 1988. A classification of the living birds of the world based on dna-dna hybridization studies. The Auk 105 ( 3): 409-423.

Siegel-Causey, D. 1997. Phylogeny of Pelecaniformes. Chap. 6. In: D. P. Mindell (Ed.) 1997. Avian Molecular Evolution and Systematics: 159-170.

Tabilo, E., R. Jorge, R. Riquelme, A. Mondaca, C. Labra, J. Campusano, M. Tabilo, M. Varela, A. Tapia \& M. Sallaberry. 1996. Management and conservation of the habitats used by migratory shorebirds at Coquimbo, Chile. Shorebird Ecology and Conservation in the Western Hemisphere. P. Hicklin Eds. International Wader Studies 8: 79-84. 
Gayana 70(1), 2006

Van Tuinen, M., D. B. Butvill, J. A. W. Kirsch \& S. B. HedGes. 2001. Convergence and divergence in the evolution of aquatic birds. Proc. R. Soc. Lond. B 268: $1345-1350$

VILINA Y. 1995. Residencia, abundancia y preferencia del hábitat del pato gargantillo (Anas Bahamensis) en el humedal "Estero El Yali", Chile Central. Anales del Museo de Historia Natural de Valparaíso 23: 89-94.

Vilina Y.A., H.L. Cofre, C. Silva-Garcia, M.F. Garcia \& C. Perez-Friedenthal. 2002. Effects of El Niño on abundance and breeding of Black- necked swans on El Yali wetland in Chile. Waterbirds. 25: 123-127.

Vilina, Y. \& V. López-Calleja. 1996. The Neotropical plovers of estero El Yali in central Chile. Shorebird Ecology and Conservation in the Western Hemisphere. P. Hicklin Eds. International Wader Studies 8: 85-92.

Ysebaert, T., P. Meininger; P. Meire; K. Devos; C. Berrevoets; R. Strucker \& E. KuiJken. 2000. Waterbird communities along the estuarine salinity gradient of the Schelde estuary, NW-Europe. Biodiversity and Conservation 9: 1275-1296.

Fecha de recepción: 02.09.05

Fecha de aceptación: 26.12.05 
Aves de aguas continentales de Chile: Victoriano, P. ET AL.

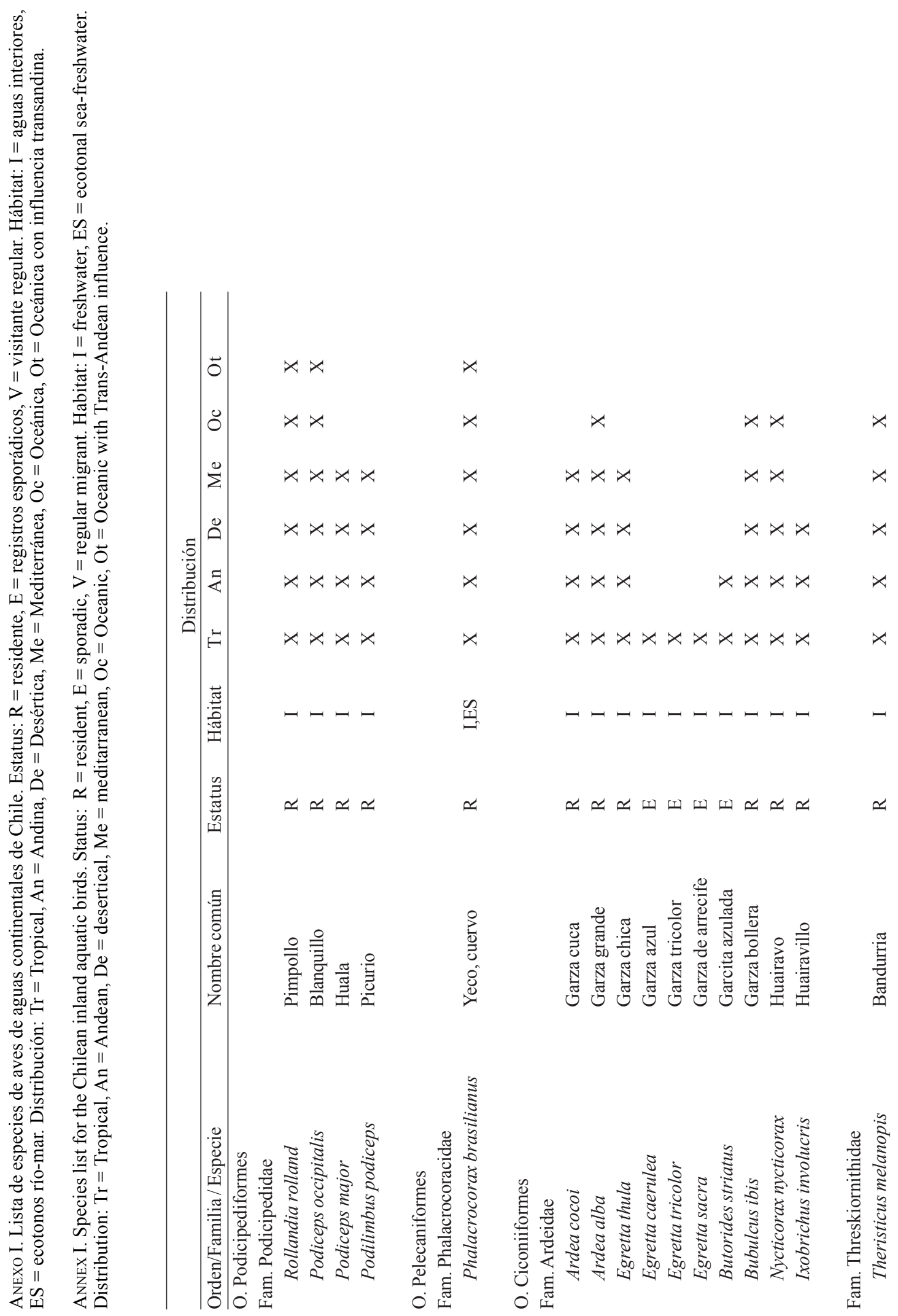


Gayana 70(1), 2006

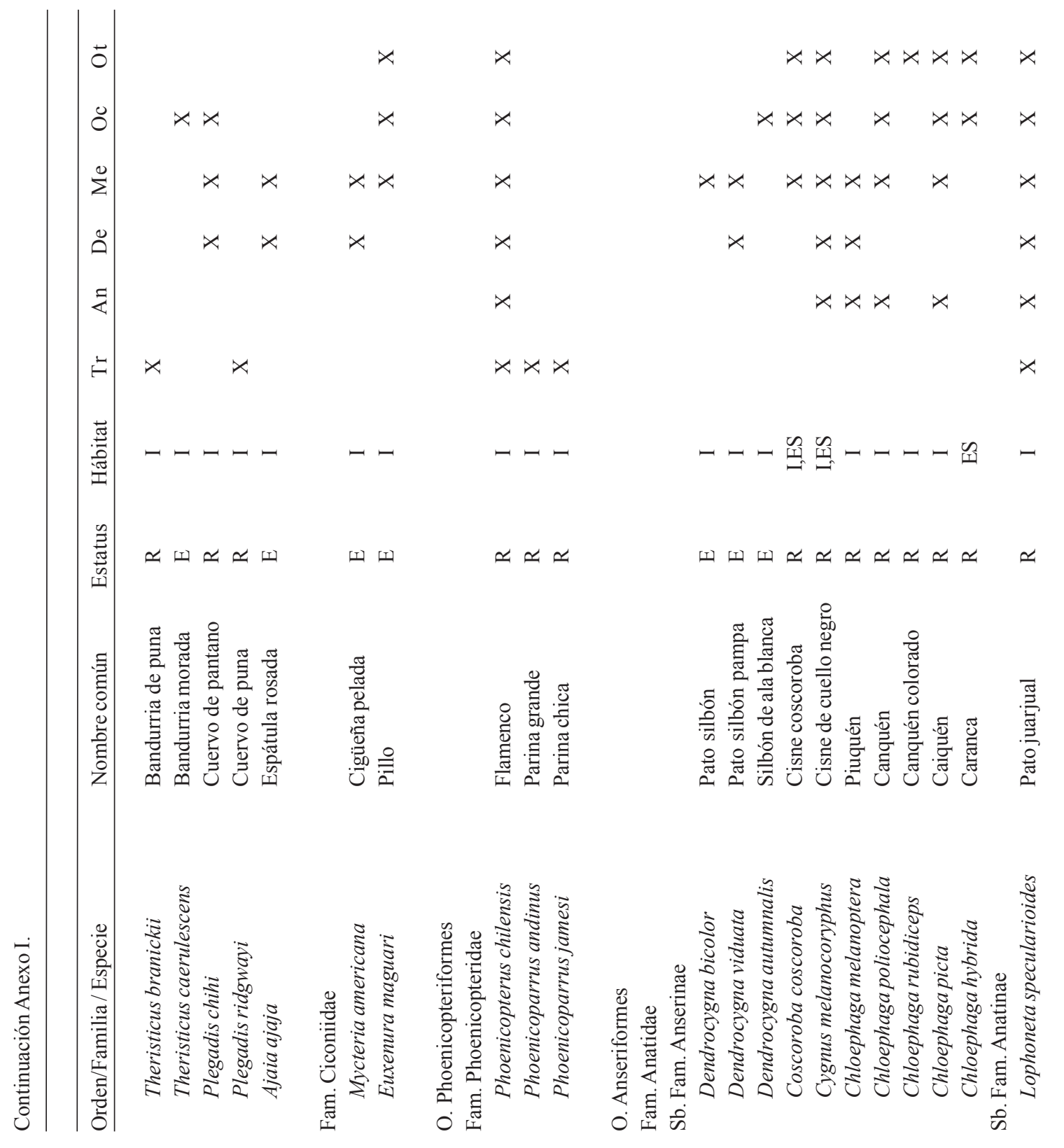


Aves de aguas continentales de Chile: Victoriano, P. ET AL.

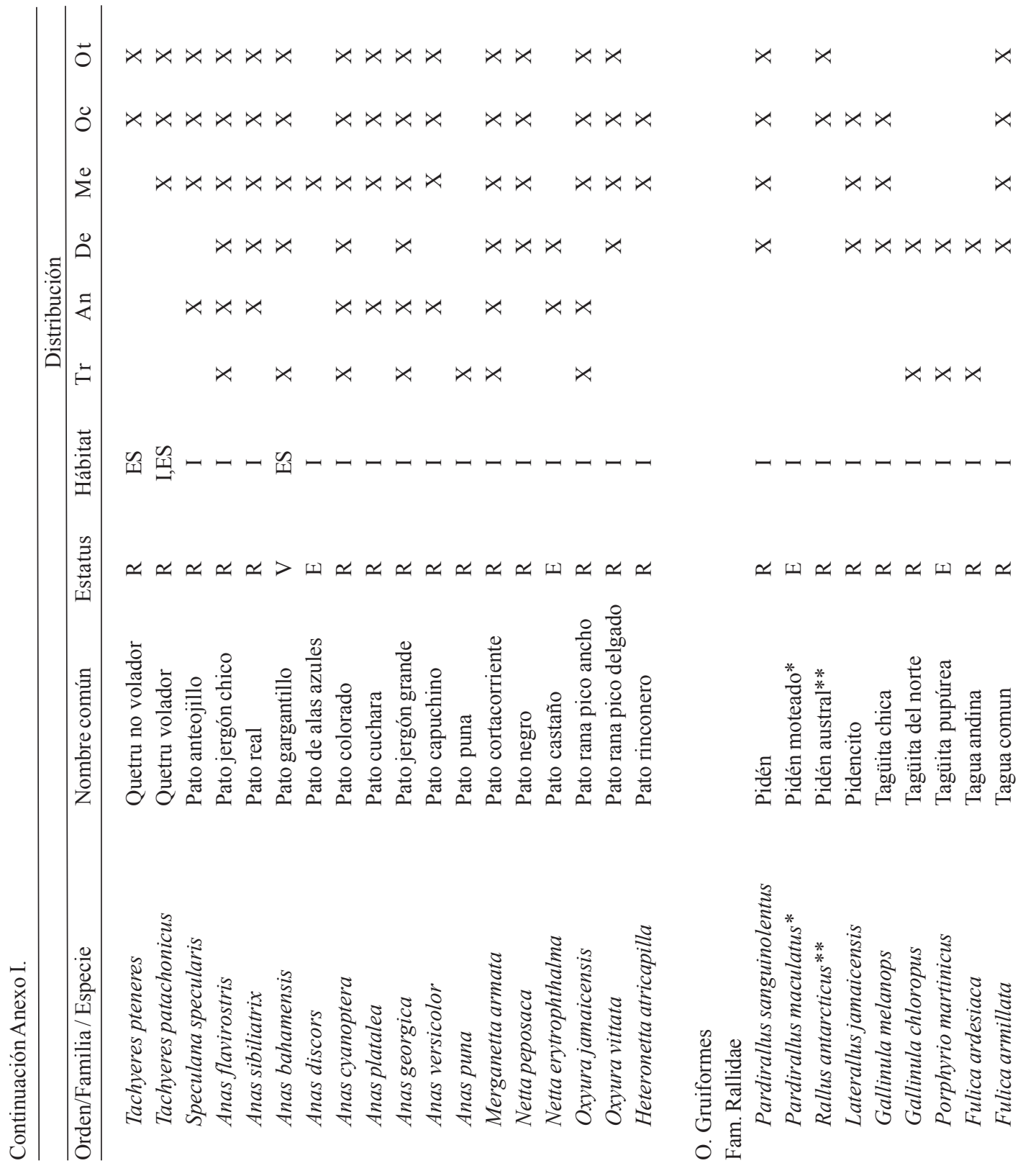




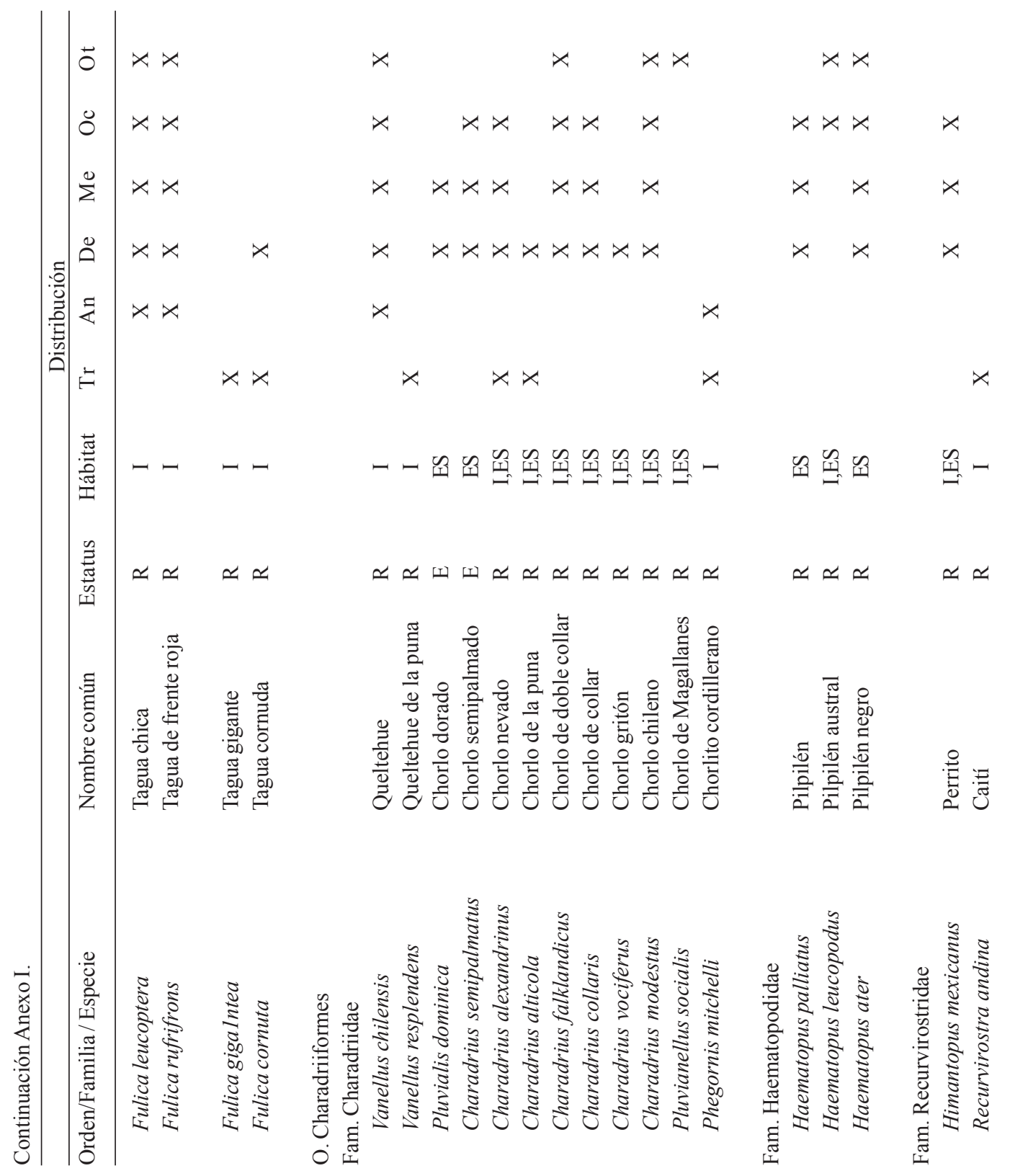


Aves de aguas continentales de Chile: Victoriano, P. ET AL.

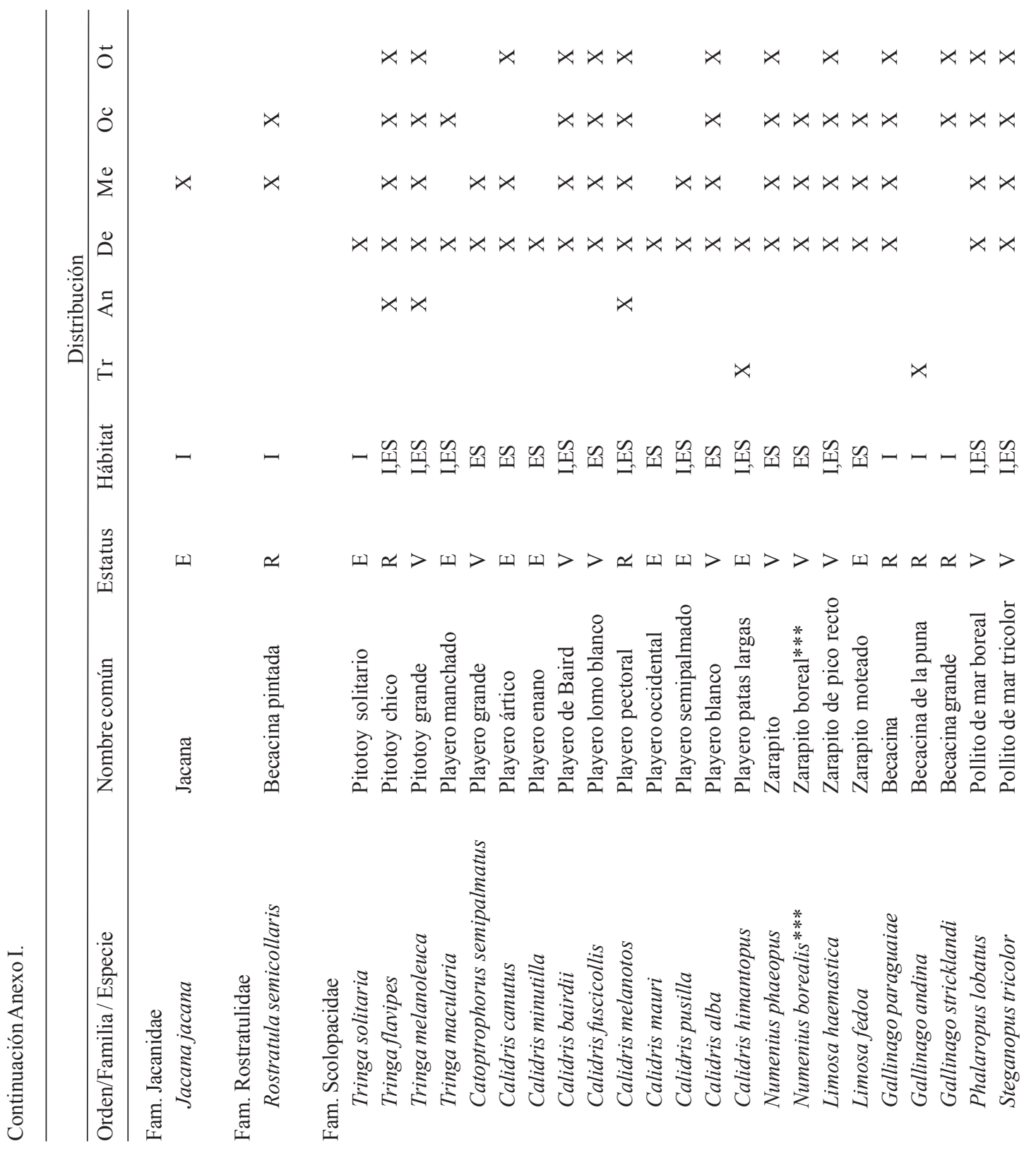


Gayana 70(1), 2006

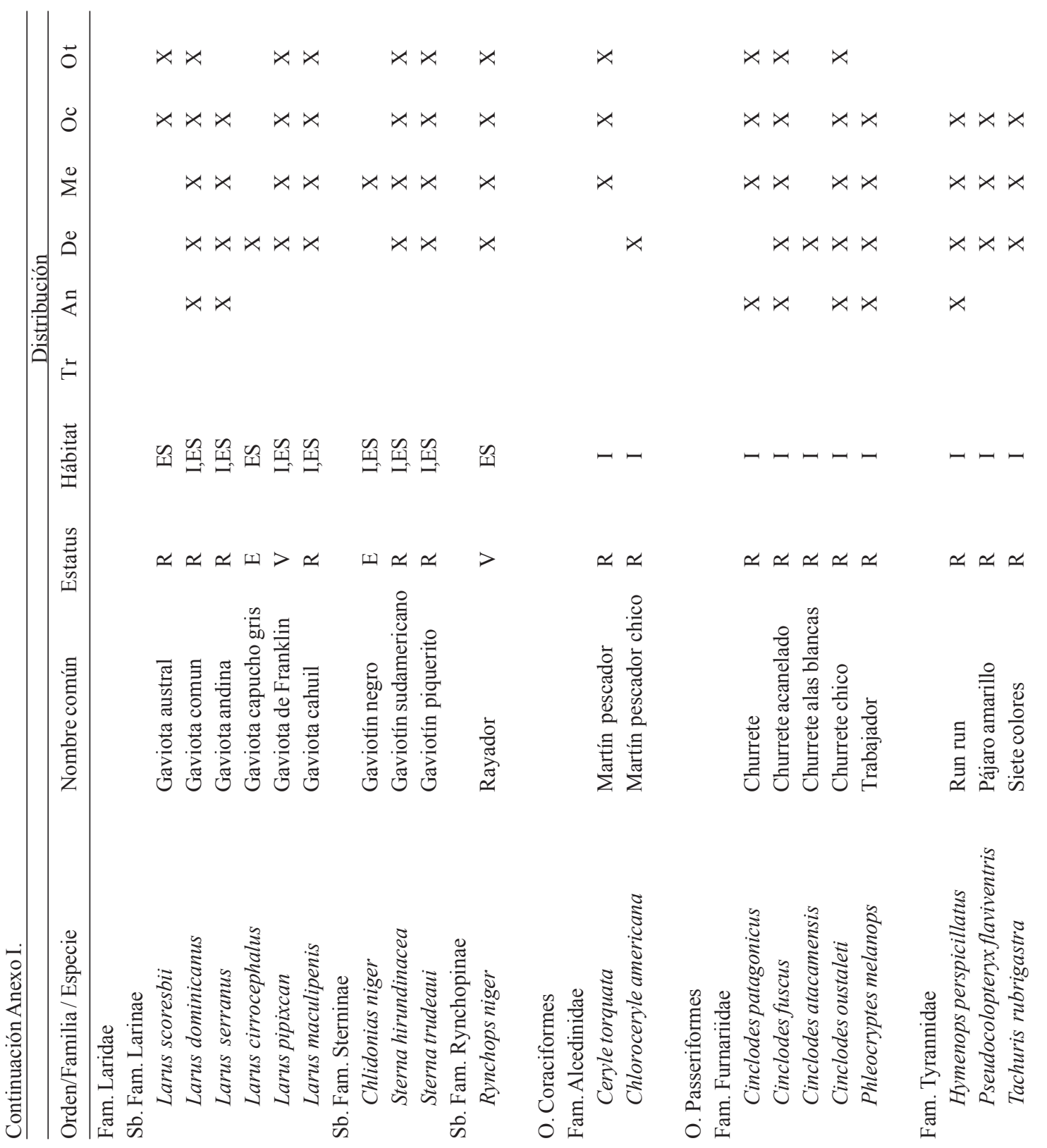


Aves de aguas continentales de Chile: Victoriano, P. ET AL.

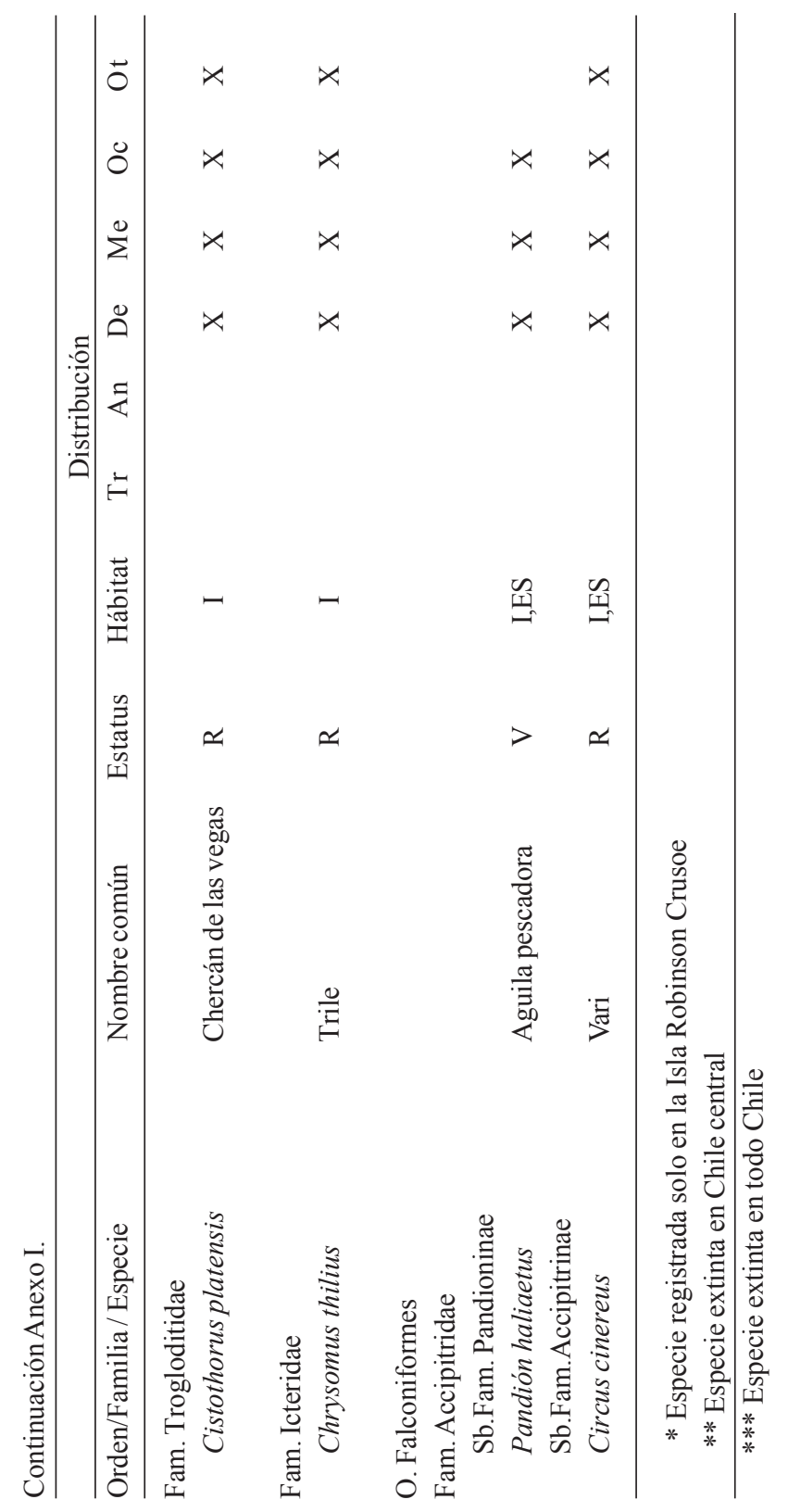


Gayana 70(1), 2006

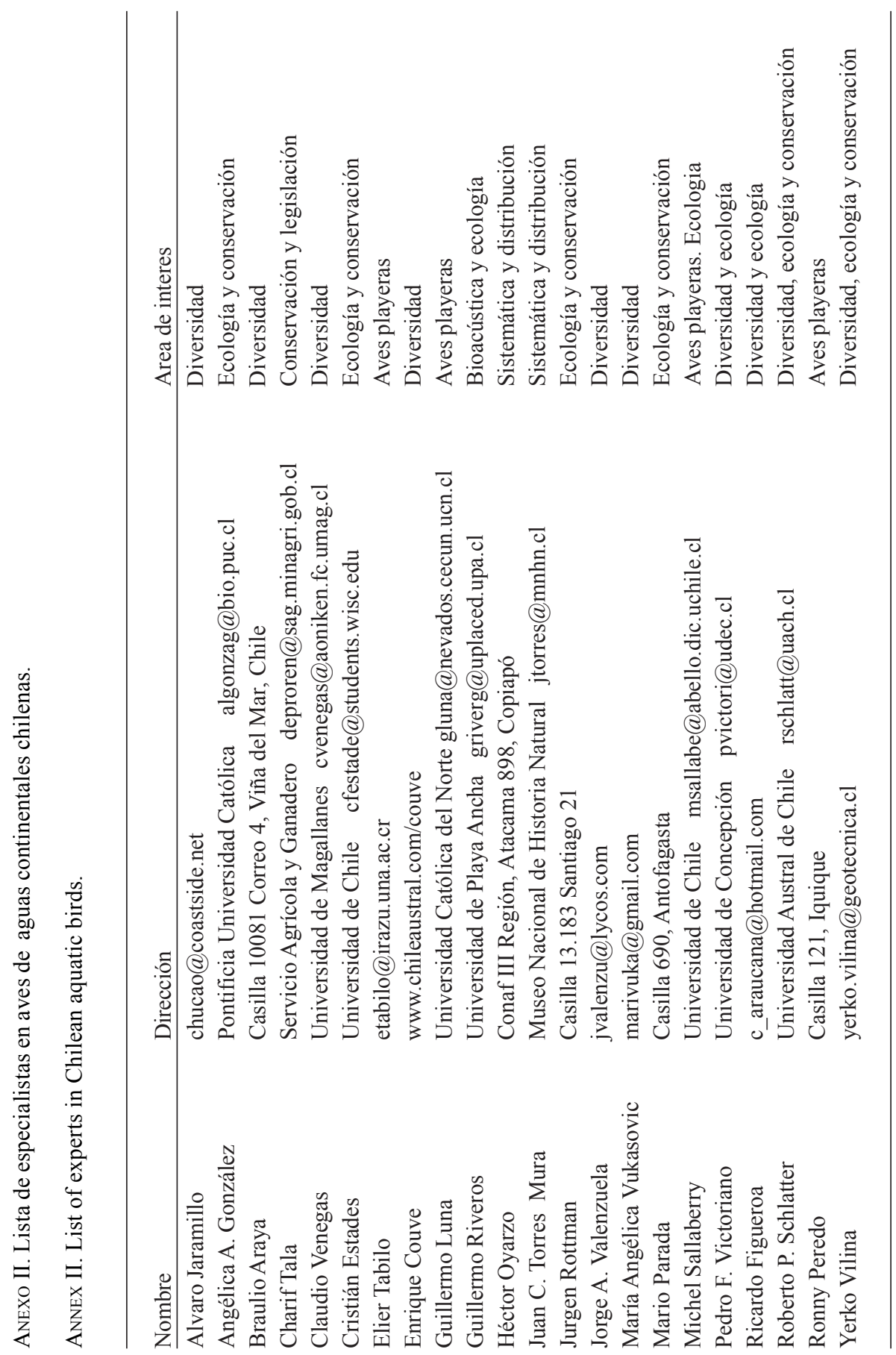

